\title{
Achados oculares em crianças de zero a seis anos de idade, residentes na cidade de São Caetano do Sul, SP
}

\author{
Ocular findings in children between 0 and 6 years of age, residing in the city of \\ São Caetano do Sul, SP
}

Sandra Maria Canelas Beer ${ }^{1}$

Marinho Jorge Scarpi ${ }^{2}$

Antonieta Antunes Minello ${ }^{3}$

\begin{tabular}{|l|}
\hline RESUMO \\
\hline Objetivo: Determinar a prevalência deerros refrativos, estrabismo, ambliopia \\
e anisometropia nas crianças pertencentes à população em estudo, utili- \\
zando dois métodos de rastreamento (triagem na escola e triagem durante \\
o dia da vacinação contra a poliomielite). Métodos: Foram examinadas 2.640 \\
crianças, residentes da cidade de São Caetano do Sul, entre zero e seis anos \\
de idade, divididas em duas amostras (A e B). A amostra A consistiu de \\
476 crianças que foram examinadas por dez oftalmologistas e onze técnicos \\
oftálmicos, durante o Dia Nacional de Vacinação (20 de junho de 1998) \\
contra a Poliomielite. A amostra B consistiu de 2.164 crianças que freqüen- \\
tavam escolas municipais, entre quatro e seis anos de idade e foram \\
examinadas por dois oftalmologistas durante o ano de 1998. Resultados: \\
Na amostra A, a prevalência de estrabismo foi de 3,36\% e de anisometropia \\
foi de1,26\%. Oprincipal tipo de erro refrativo encontrado foi a hipermetropia \\
$\leq 2 D$. Necessitaram de prescrição óptica 14,11\% das crianças. Em relação \\
à amostra B, a prevalência de estrabismo foi de 1,43\%. Nas crianças que \\
falharam na triagem, a prevalência de ambliopia foi de $1,39 \%, 2,8 \%$ de \\
anisometropia e necessitaram de prescrição óptica, 56,48\%. O principal \\
tipo de erro refrativo encontrado foi o astigmatismo hipermetrópico compos- \\
to. Conclusão: Embora em município com perfil socioeconômico pri- \\
vilegiado, a prevalência de estrabismo, anisometropia, ambliopia e erro \\
refrativo não foi inferior à relatada na literatura. Com relação àmetodologia \\
de rastreamento, o Dia Nacional de Vacinação contra a Poliomielite mos- \\
trou-se eficiente ferramenta metodológica já que houve grande participação \\
da população alvo.
\end{tabular}

Este artigo foi baseado na tese "Saúde Ocular de crianças de zero a seis anos de idade, residentes na cidade de São Caetano do Sul, SP, Brasil"; apresentada à Universidade Federal de São Paulo para obtenção do Título de Doutor em Medicina.

Trabalho oriundo do Departamento de Oftalmologia da Universidade Federal de São Paulo - UNIFESP.

Professora-colaboradora do Setor de Cirurgia Refrativa do Departamento de Oftalmologia da Universidade Federal de São Paulo - UNIFESP.

Professor assistente do Setor de Córnea do Departamento de Oftalmologia da Universidade Federal de São Paulo - UNIFESP.

${ }^{3}$ Colaborador do Setor de Glaucoma do Departamento de Oftalmologia da Universidade Federal de São Paulo - UNIFESP.

Endereço para correspondência: Sandra Maria Canelas Beer - Rua Victor Meirelles, 523 - São Caetano do Sul (SP) CEP 09581-460 E-mail: sandrabeer@uol.com.br Nota Editorial: Pela análise deste trabalho e por sua anuência na divulgação desta nota, agradecemos ao Dr. Tomás Fernando Scalamandré Mendonça.

Recebido para análise em 28.11.2001

Versão revisada recebida em 21.05.2002

Aprovação em 07.04.2003
Descritores: Triagem de massa; Ambliopia; Erros de refração; Estrabismo; Fatores socioeconômicos; Participação comunitária; Lactente; Pré-escolar; Criança

\section{INTRODUÇÃO}

As alterações oftalmológicas na idade escolar constituem uma das prioridades a considerar num programa de Saúde Escolar. Daí a importância de realizar programas de triagem visual, pelo menos no início da vida escolar, para detectar o maior número de crianças necessitadas de cuidado especializado e para que ocorra tratamento adequado para poder corrigir e/ou minimizar as doenças visuais que poderão interferir na aprendizagem ${ }^{(1)}$.

A triagem ocular já é largamente utilizada em países como Suécia, GrãBretanha e Estados Unidos ${ }^{(1-7)}$.

A Academia Americana de Oftalmologia considera que as mais sérias alterações oculares infantis, como o estrabismo, ametropias, anisometro- 
pias, cataratas congênitas, retinoblastomas, glaucoma, máformações, além de outros, podem ser descobertas pela triagem ocular na idade pré-escolar e tratadas. O principal objetivo é o tratamento precoce da ambliopia, já que ela pode ser curada e a falta de tratamento leva a um déficit permanente ${ }^{(8)}$.

A perda da capacidade visual acarreta detrimento à qualidade de vida, restrições ocupacionais, econômicas, sociais e psicológicas bem como incapacidade de trabalho e representa encargo oneroso para a sociedade $\mathrm{e}^{(9)}$.

Portanto devemos realizar ações de promoção da saúde, assim como educação em saúde para reduzir os problemas oftalmológicos que poderão comprometer o futuro das crianças ${ }^{(10)}$.

Este trabalho tem como objetivo, determinar a prevalência de erros refrativos, estrabismo, ambliopia e anisometropia, entre crianças de zero a seis anos de idade, residentes na cidade de São Caetano do Sul, São Paulo, Brasil, tendo utilizado dois métodos de rastreamento (triagem na escola e triagem durante o dia da vacinação contra a poliomielite) para traçar o perfil da saúde ocular da população em estudo.

\section{MÉTODOS}

A amostra populacional deste estudo foi constituída de crianças residentes na cidade de São Caetano do Sul, pertencente à região da Grande São Paulo. A cidade estudada apresenta-se como a cidade de maior potencial de consumo per capita do País ${ }^{(11)}$.

Foram consideradas elegíveis para o estudo crianças na faixa etária entre zero e seis anos de idade, divididas em duas amostras:

Amostra A - crianças de zero a seis anos de idade que iriam participar da campanha de vacinação contra a poliomielite a ser realizada no dia 20 de junho de 1998.

Amostra B- crianças que estudavam nas 13 Escolas Municipais Integradas (EMIs), constituída por 580 crianças (amostra B1) e nas 25 Escolas Municipais de Educação Infantil (EMEIs) constituída por 1.584 crianças (amostra B2), perfazendo um total de 2.164 crianças entre quatro anos e um mês e seis anos de idade.

O número de crianças a ser examinado no dia da vacinação contra a poliomielite foi previamente estabelecido por meio de amostragem proporcional, dividindo-se o município em cinco grandes regiões (norte, sul, leste, oeste e centro). Estabelecemos examinar 7,5 \% das crianças habitantes de cada região e para isto foi escolhido um determinado Posto de Saúde dessa região para o qual, segundo a quantidade de crianças a serem examinadas, foram destacados um a quatro oftalmologistas e um a quatro técnicos em oftalmologia para a triagem visual. A Diretoria da Saúde do município autorizou que a triagem fosse realizada no período da manhã, no dia da vacinação. Devido à cicloplegia, as filas tornaram-se enormes e muitos pais começaram a reclamar; fazendo com que encerrássemos a coleta de dados por volta das 13:00 horas. Infelizmente dois Postos de
Saúde (região Leste e Centro) não atingiram a meta de 7,5\%. Efetivamente, foram examinados $6,24 \%$ da população infantil no dia da vacinação.

Estabelecemos também que a população não deveria ficar sabendo do estudo para que a amostra fosse realmente representativa do município e não tendenciosa. Os responsáveis pelas crianças foram informados somente na hora da vacinação, da possibilidade da triagem ocular e, caso permitissem sua participação neste sentido, dariam autorização naquele momento.

Nas duas amostras foram realizados os seguintes exames oftalmológicos:

Na amostra A: anamnese dirigida, ectoscopia ocular, motricidade ocular, fundoscopia direta e refração estática.

$\mathrm{Na}$ amostra B (B1+B2): anamnese dirigida, ectoscopia ocular, motricidade ocular, acuidade visual e fundoscopia direta que foram realizados na própria escola. A refração estática, biomicroscopia e fundoscopia indireta foram realizadas no Hospital Infantil, nas crianças que foram encaminhadas por terem falhado na triagem da acuidade visual ( $\mathrm{AV} \leq 08 \mathrm{em}$ um ou dois olhos) ou que apresentaram alguma alteração ectoscópica ou astenopia.

O exame de motilidade ocular, nas duas amostras, consistiu dos testes de: “cover” alternado para perto, Hirschberg e versões, quando as crianças colaboravam. A única diferença na coleta de dados nas duas amostras foi que na amostra A o exame foi realizado por técnicos em oftalmologia, enquanto na amostra B foi realizado por médicos.

Quanto à análise estatística para estudar possíveis associações entre modalidades em relação às variáveis, usamos o teste de Qui Quadrado $\left(\chi^{2}\right)$ para tabelas de associação ou de contingência. Nas tabelas de contingência, foi utilizada a partição do Qui Quadrado. Foram sempre obedecidas as restrições de Cochran e quando estas existiam nas tabelas de associação, o teste de Qui Quadrado foi substituído pelo Teste Exato de Fischer. O nível de significância para rejeição de hipótese de nulidade foi fixado sempre em um valor igual ou menor que 0,05 (5\%). As médias foram calculadas e apresentadas a título de informação.

\section{Critérios diagnósticos}

Consideramos os seguintes critérios para diagnosticar as afecções oculares encontradas nesta pesquisa.

Ametropia ou erro refrativo - qualquer erro refracional maior ou igual a $0,5 \mathrm{D}$.

Ambliopia - déficit funcional com a melhor correção óptica de acuidade visual (na amostra B com o uso da correção adequada durante o exame refracional) sem causa orgânica, levando a uma diferença de uma ou mais linhas na tabela de Snellen, quando comparamos os dois olhos. Considerarmos as formas anisometrópica, refracional, estrábica e por privação.

Estrabismo - desalinhamento evidente do eixo ocular.

Anisometropia - diferença de uma ou mais dioptrias, tanto esférica como cilíndrica, entre a refração dos dois olhos. 
Na amostra B, somente os indivíduos que apresentavam $\mathrm{AV}<0,8$ em um dos olhos, foram encaminhados para o exame oftalmológico completo, portanto o restante não necessitava de correção óptica.

\section{RESULTADOS}

\section{Amostra A}

Foram avaliadas 476 crianças de zero a seis anos que participaram da campanha de vacinação contra a poliomielite. A ortotropia foi o principal achado (398 crianças - 83,61\%). Quanto à presença de desvio, encontramos 16 casos de estrabismo (3,36\%), sendo quatro casos de esotropia, nove casos de exotropia e três casos de exotropia intermitente. Em relação à foria, a exoforia foi predominante (58 casos - 93,55\%).

$\mathrm{Na}$ tabela 1, mostramos os achados das afecções oculares encontradas na amostra A, considerando cada criança como dois olhos isoladamente (exceto para estrabismo e anisometropia, onde consideramos a criança como única).

Das primeiras 476 crianças que fizeram o exame de motricidade ocular, apenas 397 crianças, tiveram autorização de seus responsáveis para realizar cicloplegia e retinoscopia na avaliação do erro refracional.

Os principais erros refracionais encontrados foram: hipermetropia $\leq 2 \mathrm{D}$ (55,92\%), seguida de emetropia (14,23\%) e astigmatismo hipermetrópico composto (12,85\%).

A prevalência de estrabismo foi de 3,36\% e a de anisometropia, $1,26 \%$.

Das crianças cicloplegiadas ( $\mathrm{N}=397)$, apenas $56(14,11 \%)$ apresentaram ametropia e necessitaram de correção óptica.

Na amostra A, encontramos 83,38\% das crianças com equivalente esférico entre -0,5D e +1,5D. A média do equivalente esférico da população estudada foi de 1,18.

Das 142 crianças com astigmatismo,131 (92,25\%) apresentaram astigmatismo a favor da regra, 6 (7,04\%) astigmatismo contra a regra e 5 (3,52\%) astigmatismo oblíquo.

\section{Amostra B}

A amostra B consistiu em 2.164 crianças, sendo que 580 pertenciam às EMIs e 1.584 às EMEIs. Na amostra B, 2.132 $(98,52 \%)$ crianças apresentaram ortotropia.

A prevalência de estrabismo foi de 1,43\% (31 crianças) sendo 19 crianças com esotropia,11 com exotropia e uma criança com esotropia intermitente.

Em relação ao erro refrativo, foram encaminhadas para exame oftalmológico completo 450 crianças que apresentaram $\mathrm{AV} \leq 0,8$, em um e/ou nos dois olhos. Apenas 216 crianças compareceram para esse exame. Encontramos 12 crianças com algum tipo de anisometropia e 30 apresentaram ambliopia. Requereram correção óptica 122 crianças das quais foram examinadas. Na tabela 2 mostramos as afecções oculares encontradas nas crianças que foram cicloplegiadas na amostra B, considerando cada criança com dois olhos isoladamente (ex-

\begin{tabular}{|c|c|c|c|}
\hline \multirow{3}{*}{$\begin{array}{l}\text { Afecções oculares } \\
\text { Amostra A } \\
\text { Estrabismo }\end{array}$} & \multicolumn{3}{|c|}{ Número de crianças } \\
\hline & \multicolumn{3}{|c|}{ Feminino \% Masculino \% Total \% } \\
\hline & $1,26(6)$ & $2,10(10)$ & $3,36(16)$ \\
\hline Anisometropia & $0,25(1)$ & $1,01(4)$ & $1,26(5)$ \\
\hline Refração plana & $6,55(52)$ & $7,68(61)$ & $14,23(113)$ \\
\hline Hipermetropia >2 D & $4,91$ ( 39$)$ & $4,16(33)$ & $9,07(72)$ \\
\hline Hipermetropia $\leq 2 \mathrm{D}$ & $30,60(243)$ & $25,31(201)$ & $55,92(444)$ \\
\hline $\begin{array}{l}\text { Astigmatismo } \\
\text { hipermetrópico simples }\end{array}$ & $1,26(10)$ & $2,27(18)$ & $3,53(28)$ \\
\hline $\begin{array}{l}\text { Astigmatismo } \\
\text { hipermetrópico composto }\end{array}$ & $6,17(49)$ & $6,67(53)$ & $12,85(102)$ \\
\hline $\begin{array}{l}\text { Astigmatismo } \\
\text { miópico simples }\end{array}$ & $0,50(4)$ & $0,25(2)$ & $0,75(6)$ \\
\hline $\begin{array}{l}\text { Astigmatismo } \\
\text { miópico composto }\end{array}$ & $0,25(2)$ & $0\left(\begin{array}{l}0 \\
0\end{array}\right.$ & $0,25(2)$ \\
\hline Astigmatismo misto & $0,25(2)$ & $0,25(2)$ & $0,5(4)$ \\
\hline Miopia & $0,63(5)$ & $2,27(18)$ & $2,90(23)$ \\
\hline $\begin{array}{l}\left(^{*}\right) \text { Mais de um diagnóstico po } \\
N=476 \text { crianças (estrabismo) } \\
N=397 \text { crianças (anisometrop } \\
N=794 \text { olhos (erros refraciona }\end{array}$ & $\begin{array}{l}\text { or criança } \\
\text { pia) } \\
\text { lal) }\end{array}$ & & \\
\hline
\end{tabular}

\begin{tabular}{|c|c|c|c|}
\hline \multirow{2}{*}{$\begin{array}{l}\text { Afecções oculares } \\
\text { Amostra B }\end{array}$} & \multicolumn{3}{|c|}{ Número de crianças } \\
\hline & Feminino \% & Masculino & $\%$ Total \% \\
\hline Estrabismo & $0,88(19)$ & $0,55(12)$ & $1,43(31)$ \\
\hline Anisometropia & $0,42(9)$ & $0,14(3)$ & $2,8 \quad(12)$ \\
\hline Ambliopia & $0,97(21)$ & 0,42 ( 9) & $6,9(30)$ \\
\hline Refração plana & $0,93(4)$ & $0,23(1)$ & $1,16(5)$ \\
\hline Hipermetropia >2 D & $3,94(17)$ & $5,09(22)$ & $9,03(39)$ \\
\hline Hipermetropia $\leq 2 \mathrm{D}$ & $14,58(63)$ & $15,28(66)$ & $29,86(129)$ \\
\hline $\begin{array}{l}\text { Astigmatismo } \\
\text { hipermetrópico simples }\end{array}$ & $3,47(15)$ & $3,01(13)$ & $6,48(28)$ \\
\hline $\begin{array}{l}\text { Astigmatismo } \\
\text { hipermetrópico composto }\end{array}$ & $13,43(58)$ & $17,36(75)$ & $30,79(133)$ \\
\hline $\begin{array}{l}\text { Astigmatismo } \\
\text { miópico simples }\end{array}$ & 3,24 (14) & $5,09(22)$ & $8,33(36)$ \\
\hline $\begin{array}{l}\text { Astigmatismo } \\
\text { miópico composto }\end{array}$ & $1,62(7)$ & $0,46(2)$ & $2,08(9)$ \\
\hline Astigmatismo misto & $2,77(12)$ & $4,17(18)$ & $6,94(30)$ \\
\hline Miopia & $3,24(14)$ & $2,08$ ( 9$)$ & $5,32(23)$ \\
\hline $\begin{array}{l}\text { ( }) \text { Mais de um diagnóstico por } \\
\mathrm{N}=216 \text { crianças ( } 432 \text { olhos) pa } \\
\mathrm{N}=216 \text { crianças para anisome } \\
\mathrm{N}=2164 \text { crianças para estrabi }\end{array}$ & $\begin{array}{l}\text { or criança } \\
\text { para erro refrativ } \\
\text { etropia e ambliop } \\
\text { jismo }\end{array}$ & & \\
\hline
\end{tabular}

ceto para estrabismo, onde consideramos todas as crianças que foram triadas).

Caso considerássemos que as outras 234 crianças que foram encaminhadas e faltaram no dia do exame completo apresentassem as mesmas prevalências das crianças examinadas, $11,74 \%$ das crianças da amostra B receberiam prescrição óptica. A prevalência de anisometropia seria de 1,15\% e a prevalência de ambliopia de 2,88\%. 
Quanto aos erros refracionais detectados na amostra B, encontramos principalmente astigmatismo hipermetrópico composto, representando 30,79\% dos casos, seguidos por hipermetropia $\leq 2 \mathrm{D}$, representando $29,86 \%$ dos casos.

Em relação ao tipo de astigmatismo que encontramos na amostra B, 91,9\% das crianças apresentaram astigmatismo a favor da regra.

Em relação ao equivalente esférico encontrado na amostra B1, 68,57\% dos olhos das crianças apresentaram equivalente esférico entre -0,5 e +1,5 D. A média do equivalente esférico da população (B1) estudada foi de 1,71 .

Em relação à amostra B2, 79,39\% dos casos apresentaram equivalente esférico entre-0,5 D e +1,5 D. A média do equivalente esférico da população estudada (B2) foi de 1,35 e a média do equivalente esférico da amostra B total foi de 1,46.

Para que pudéssemos efetuar uma comparação entre as duas amostras e realizar a análise estatística para saber se havia alguma diferença relevante, foram consideradas apenas as crianças da amostra A com quatro anos ou mais (124 crianças).

Não encontramos diferença significante quando realizamos a comparação entre as duas amostras, A e B, nas seguintes afecções: estrabismo, anisometropia, hipermetropia $>2$, astigmatismo hipermetrópico simples, miopia e astigmatismo miópico composto.

Todavia quando comparamos as duas amostras no que concerne às afecções: refração plana, hipermetropia $\leq 2 \mathrm{D}$, astigmatismo hipermetrópico composto, astigmatismo miópico simples e astigmatismo misto, detectamos diferença estatisticamente significante.

\section{DISCUSSÃO}

No presente trabalho detectamos 2.530 (95,83\%) crianças com ortotropia como o principal achado. Em relação à prevalência de foria foi de apenas 2,38\% (63 crianças), sendo 58 crianças portadoras de exoforia e quatro de esoforia na amostra A e apenas uma de esoforia na amostra B. Tais achados coincidem com os encontrados em uma triagem realizada na cidade de Paulínia, interior de São Paulo, onde foram avaliadas 569 crianças, entre dois e nove anos de idade e registradas $58,7 \%$ de ortotropia e entre as forias $92,1 \%$ eram exoforia ${ }^{(12)}$. Em relação à foria, a diferença apresentada entre a amostra A e B, talvez deva-se ao fato que os testes de versões, "cover" simples alternado para perto terem sido aplicados por técnicos em oftalmologia apenas na amostra A. Não realizamos o teste de “cover” a distância devido à dificuldade em conseguirmos a atenção e cooperação das crianças na fixação de um objeto para longe em um ambiente tumultuado como um Posto de Saúde no dia de vacinação, além do mais algumas crianças eram bem pequenas e não colaborativas. Possivelmente, se o teste de "cover " simples alternado a distância tivesse sido realizado e aplicado por técnicos em oftalmologia nas duas amostras, talvez tivéssemos tido um melhor perfil da motricidade ocular.

A maioria dos trabalhos refere uma prevalência de estra- bismo na população caucasiana em torno de $1-4 \%^{(13-14)}$. Na população em estudo, encontramos na amostra A uma prevalência de estrabismo de 3,36\% e na amostra B, de 1,43\%. No cômputo geral a prevalência de estrabismo na cidade de São Caetano do Sul foi de 1,78\% (47 crianças). As prevalências foram diferentes, possivelmente porque a amostra B possuia maior número de crianças entre um e quatro anos de idade, mas quando comparamos a amostra A com amostra B, levando em conta a idade, considerando, portanto apenas as crianças com mais de quatro anos (124 crianças) da amostra A, a diferença não foi significante. Novamente nossos achados coincidem com os encontrados no trabalho de Costa e col, $1979^{(12)}$, realizado em outra cidade do interior de São Paulo, onde a prevalência de estrabismo foi de 1,58\%.

Neste estudo, as amostras A e B não apresentaram diferenças estatisticamente significantes no que tange à prevalência do número de casos de estrabismo observado próximo aos quatro anos de idade; achado este concordante com a literatura, onde se relata que a maioria de casos de estrabismo ocorre entre um e quatro anos de idade ${ }^{(13)}$.

Em relação ao tipo de estrabismo, na amostra A houve maior prevalência de exotropia (56,25\% - 9 casos) e na amostra B houve predominância de esotropia (61,29\% -19 casos). Na amostragem geral encontramos uma prevalência de esotropia (48,93\% - 23 casos) próxima da prevalência da exotropia (42,55\% - 20 casos). Encontramos também 8,51\% de estrabismo intermitente, sendo três casos de $\mathrm{X}(\mathrm{T})$ e um caso de $\mathrm{E}(\mathrm{T})$. Outro achado foi de hipertropia associada à ET (dois casos) e um caso associado à XT.

Nossos achados diferiram daqueles registrados na literatura compulsada, pois a esotropia é geralmente três vezes mais freqüente do que a exotropia. Ademais, houve maior incidência de casos de estrabismo intermitente ${ }^{(12,15)}$. A explicação possível para os nossos achados deve-se provavelmente a um erro na aplicação da metodologia de avaliação da motilidade ocular ou mesmo ao acaso.

Em relação à prevalência de anisometropia encontrada na população estudada, o achado foi de $1,26 \%$ na amostra A e na amostra B de 1,15\% (considerando a mesma prevalência de casos nas outras crianças que não compareceram ao exame oftalmológico completo).

Os achados da literatura variam em relação à prevalência da anisometropia. Em 1977, realizaram uma triagem em 1.400 escolares, entre seis e quinze anos de idade, referindo uma prevalência de 2,36\% de anisometropia (diferença de 2,0 D esféricas ou EE entre os dois olhos) ${ }^{(16)}$. Em outra realizada em 1979 , referiram a taxa de $1,23 \%$, sendo que os autores consideraram anisometropia como a diferença entre dois olhos de uma dioptria esférica ou duas dioptrias cilíndricas ${ }^{(12)}$.

A ambliopia só foi estudada na amostra B (não foi medida AV na amostra A), onde encontramos uma prevalência de 30 casos entre os pacientes que foram cicloplegiados. Hipoteticamente, caso considerássemos a mesma prevalência nas outras crianças que foram encaminhadas e não compareceram, teríamos uma prevalência de 2,88 \% na amostra B. 
A prevalência de ambliopia varia de $1-5 \%{ }^{(8)}$ na literatura ${ }^{(17)}$. Foi realizado um estudo com 5.329 crianças, divididas em cinco grupos, pela faixa etária, e foi determinada a prevalência de ambliopia em cada grupo ${ }^{(17)}$. Esta foi crescente conforme aumentou a faixa etária, variando de $0,4 \%$ até $2,1 \%$ nos grupos de crianças com maior idade. A prevalência média foi de 1,2\%. Outro trabalho referiram uma taxa de 3,9\%(14).

Em relação aos erros refrativos encontrados na amostra A, $14,11 \%$ das crianças apresentaram alguma ametropia que necessitou de correção óptica e na amostra B, apenas 48\% (216 crianças) das crianças encaminhadas para o exame oftalmológico completo compareceram, dentre elas, 56,48\% necessitaram de correção óptica.

A literatura refere que aproximadamente $10 \%$ das crianças nessa faixa etária têm prescrição óptica, como citado no estudo de Köhler e Stigmar ${ }^{(4)}$, que realizaram triagem em 2.447 crianças com quatro anos de idade e constataram a necessidade de prescrever óculos para 8,0\% da população estudada. Em outro estudo foi mencionada uma taxa de 6,33\% de prescrição de óculos ${ }^{(12)}$.

$\mathrm{Na}$ amostra $\mathrm{A}$, os principais erros refrativos encontrados foram a hipermetropia $\leq 2 \mathrm{D}$, seguida por refração plana e astigmatismo hipermetrópico composto. Na amostra B, encontramos principalmente astigmatismo hipermetrópico composto, seguido de perto por hipermetropia $\leq 2 \mathrm{D}$. Estes achados estão em concordância com a literatura que relata, nos primeiros anos de vida, a baixa hipermetropia como o principal erro refrativo encontrado ${ }^{(4,18-19)}$.

Em relação ao equivalente esférico, a média do equivalente esférico da amostra A foi de 1,18 e da amostra B foi de 1,46; portanto os dados são bastante parecidos com os da literatura, onde a distribuição da população em relação ao equivalente esférico é bastante semelhante à verificada em nosso estudo $^{(20-21)}$.

Quando as duas amostras foram comparadas, notamos que algumas ametropias apresentaram diferenças significantes, como a hipermetropia $\leq 2 \mathrm{D}$, mas quando as comparamos, segundo a idade, observamos que a prevalência desta ametropia era decrescente conforme o aumento da idade, todavia os achados de ambas as amostras quando comparadas entre si não eram significantes. Na comparação das amostras A e B, em relação à presença de hipermetropia $\leq 2 \mathrm{D}$, notamos sua maior prevalência na amostra A. Acreditamos que isso ocorreu porque na amostra $A$ todos os pacientes triados foram cicloplegiados e na amostra B apenas os que apresentavam AV inferior a 0,8, havendo portanto um menor número de casos de hipermetropias $\leq 2 \mathrm{D}$, já que ametropia em crianças geralmente não causa baixa $\mathrm{AV}$, razão pela qual não foram enviados para o exame oftalmológico completo. Este fato deve ser considerado para não haver erro de interpretação.

Notamos uma diferença bastante significante no astigmatismo hipermetrópico em relação à presença ou ausência de ametropia. Consideramos mais uma vez que o acaso foi responsável pela maior prevalência na amostra B.

A prevalência de astigmatismo miópico simples, quando analisamos as amostras em relação à presença ou ausência de ametropia, foi altamente significante. Acreditamos que isso se deva ao fato de que a amostra $\mathrm{B}$ apresentava um maior número de crianças mais velhas, portanto levando em consideração que há uma tendência à miopização com o passar da idade, isto explicaria a maior incidência desse tipo de astigmatismo ${ }^{(19,22-23)}$.

$\mathrm{O}$ astigmatismo misto apresentou, em relação à presença ou ausência de ametropia, uma diferença estatística significante. Não conseguimos uma explicação razoável para o fato, a não ser o acaso ou talvez o tamanho da amostra.

Neste estudo, o astigmatismo a favor da regra foi o principal achado. Em relação à literatura, nossos achados são semelhantes, caso consideremos que a grande parte da população estudada tem mais de quatro anos de idade, (principalmente aquela da amostra B), pois a maioria dos autores relata maior predominância do tipo "a favor da regra” após os quatro primeiros anos de vida, e o tipo "contra a regra " nos primeiros anos de vida. Vários estudos concordam que os astigmatismos oblíquos são mais $\operatorname{raros}^{(21,24-26)}$.

Sob o ponto de vista epidemiológico, é importante ressaltar que a ambliopia meridional é causada pela privação devida à presença de astigmatismo no meridiano mais hipermétrope. Este meridiano provavelmente fica borrado e leva à ambliopia. Quando associada à anisometropia, a ambliopia é mais freqüente. ${ }^{(27)}$ Este fato torna importante a avaliação precoce do astigmatismo para diminuir a gravidade da ambliopia causada e possibilitar sua eliminação, já que este é o tipo de ambliopia de melhor prognóstico.

A triagem ocular tem duas justificativas fundamentais para continuar existindo: em níveis de saúde pública e no plano educacional ${ }^{(28)}$.

No aspecto de saúde pública, a triagem é necessária porque um grande número de crianças chega à idade escolar sem nunca ter passado por um exame oftalmológico. Pesquisas de saúde ocular apontam que em torno de $15 \%$ das crianças da primeira série possuem alguma alteração visual e apenas $20 \%$ dessas crianças são acompanhadas por médicos ${ }^{(28)}$.

Idealmente todas as crianças deveriam ser examinadas por um oftalmologista, antes de iniciarem a vida escolar, e acompanhadas no decorrer dos anos. No nosso país onde o número de médicos especialistas é pequeno na maioria das regiões, excluindo as grandes metrópoles, além da dificuldade de acesso da população carente ao serviço médico, são esperados taxas ainda maiores de crianças que nunca tenham sido avaliadas por um oftalmologista.

Portanto as triagens realizadas por universidades, sociedades de classe, associações filantrópicas, governo, entre outros, contando com o trabalho voluntário de especialistas, alunos, profissionais da saúde, seriam um modo de preencher essa lacuna da saúde pública em nosso país; pois além de ser uma forma mais barata, é um eficiente método de identificação de crianças com grande risco para alterações visuais. No ponto de vista de saúde pública, a única ressalva é que seus programas têm uma certa periodicidade para que haja acompanhamento para os casos identificados, assim como a identificação de casos novos a cada ano. 
Em relação ao plano educacional, sabemos que uma boa visão é vital para o melhor desempenho do indivíduo em vários níveis. Ela está intimamente relacionada com o ato de aprendizagem e, como foi exposto anteriormente, a grande maioria das crianças chega à idade escolar sem nunca ter passado por um exame oftalmológico; portanto, caso essas crianças não sejam identificadas e tratadas, elas poderão ter um desempenho escolar deficiente e não atingir o potencial máximo intelectual. Assim sendo a triagem desempenha um importante papel na identificação dessas crianças de risco.

Pelo exposto, a triagem é um método válido, pois nos leva a interferir no prognóstico acuidade visual final do indivíduo, já que a detecção precoce de altos erros refracionais, quando prescritos parcialmente, não interrompe o processo de emetropização e leva à boa acuidade visual final ${ }^{(29)}$. Outro fator favorável seria a prescrição precoce da anisometropia, que preveniria a ambliopia e o prognóstico da acuidade visual final. Devemos lembrar também que, como existe um alto número de casos de astigmatismo, a prevenção da ambliopia meridional também é um fator favorável. Embora a triagem favoreça a detecção precoce de ambliopia e estrabismo, ela não interfere na diminuição da prevalência destas doenças, como foi relatado no trabalho de Ingram e $\operatorname{col}^{(29)}$, onde os autores mostraram que a prevalência de estrabismo e ambliopia não diminuiu com a prescrição de óculos aos seis meses de idade, mas a acuidade visual final era melhor do que nas crianças que não usaram refração e que não foram submetidas a tratamento.

Os achados das afecções visuais da população estudada mostraram-se semelhantes aos achados da literatura. Embora São Caetano do Sul possua um elevado poder aquisitivo, a população infantil apresentava-se praticamente desassistida na área oftalmológica, já que a maioria das crianças examinadas nunca tinha passado por uma avaliação oftalmológica e muitos de seus problemas foram descobertos por meio da triagem. Realizada neste estudo. Infelizmente isto é uma realidade em nosso país, onde a maioria das crianças só é levada à assistência oftalmológica tardiamente, quando apresentam sintomas de baixa AV ou até mesmo cegueira e, nesse momento, o tratamento já não apresenta o resultado desejado. Este fato denota a carência de cuidados médicos e desinformação não apenas da população, mas também da classe dos médicos pediatras, que só encaminham para os oftalmologistas casos francamente patológicos e nunca sugerem uma avaliação oftalmológica rotineira como é preconizado pela Academia Americana de Oftalmologia ${ }^{(8)}$.

A campanha de vacinação contra a pólio mostrou-se uma excelente oportunidade para a coleta de dados sobre a saúde ocular de crianças de baixa idade, já que houve uma grande participação da população alvo. Na campanha realizada em junho de 1998, houve a participação de 7.629 crianças entre zero e seis anos de idade, representando $90,77 \%$ da população infantil desta faixa etária do município de São Caetano do Sul (dados fornecidos pelo DIR- Direção Regional de Saúde). Portanto o Dia Nacional da Vacinação contra a Pólio mostrouse uma excelente ferramenta metodológica para o rastreamen- to de doenças infantis, não apresentando nenhuma diferença significante em relação à triagem em escolas, ferramenta classicamente conhecida, talvez até mais representativa, já que há praticamente $100 \%$ da população. Em relação à metodologia aplicada, o único ponto desfavorável foi o tempo de espera para a cicloplegia ser adequada, que fez com que atrasássemos um pouco o andamento normal da vacinação, mas com maior freqüência desse tipo de triagem, certamente a população compreenderá melhor a sua importância.

\section{CONCLUSÃO}

O perfil dos distúrbios visuais da cidade de São Caetano do Sul foi traçado, mostrando a necessidade de que atitudes sejam tomadas por autoridades, médicos oftalmologistas, médicos pediatras e professores da cidade para mudar esse perfil, pois a cidade embora apontada há vários anos como a cidade modelo, não apresenta praticamente nenhum programa de saúde pública voltado para a saúde visual das crianças a não ser o atendimento primário no Hospital infantil

Em dezembro de 1999, foi promulgada pela Câmara Municipal da cidade de São Caetano do Sul a Lei Municipal (lei 3862 /28 de dezembro de 1999) que torna obrigatória a avaliação oftalmológica anual das crianças de três a seis anos de idade, freqüentadoras das EMIs e EMEIS.

Esperamos que este seja o primeiro passo para a melhoria da Saúde Ocular da população por nós estudada e que novas ações em saúde pública ocorram.

\section{AGRADECIMENTOS}

Aos amigos, doutores: Cínthia Amorim, Daniela Fairbanks, Elizabeth Martins Nogueira, Lênio Alvarenga, Maria Cristina Leoratti, Maria Regina Chalita, Namir dos Santos e técnicos em oftalmologia que participaram da coleta de dados, pela colaboração e espírito de equipe indispensáveis à realização desta pesquisa.

\section{ABSTRACT}

Purpose: To establish the prevalence of refractive errors, strabismus, amblyopia and anisometropia, among children in a population, using two methods of screening (at school and at the National Polio Vaccination Day). Methods: We examined 2,640 children, between 0 and 6 years of age. The children, residents of the city São Caetano do Sul, were divided into two groups (A and B). Group A: 476 children, with an age range of a few months to 6 years, had their eyes examined by 10 ophthalmologists and 11 ophthalmology technicians, during the National Polio Vaccination Day (June 20,1998). Group B: 2,164 children attending municipal schools (EMEIs and EMIs), between 4 and 6 years of age, who had their eyes examined by two ophthalmologists during the year of 1998. Results: In group A, the prevalence of strabismus was 3.36\% and that of 
anisometropia was $1.26 \%$. The most common type of refractive error was hypermetropia $\leq 2 \mathrm{D}$ and $14.11 \%$ required optical prescription. In group $\mathrm{B}$, the prevalence of strabismus was $1.43 \%$. In the group who failed in the screening, the prevalence of amblyopia was $1.39 \%$ and that of anisometropia was $2.8 \%$. The most common type of refractive error found was hypermetropic astigmatism and 56.48\% required optical prescription. Conclusion: The prevalence of the ocular findings in children in São Caetano do Sul, although being a city with a privileged socioeconomic profile, is similar to that reported in the literature. Ophthalmic examination on National Polio Vaccination Day showed to be an efficient screening tool.

Keywords: Mass screening; Amblyopia; Refractive errors; Strabismus; Socioeconomic factors; Consumer participation; Infant; Child, preschool; Child

\section{REFERÊNCIAS}

1. Simons. Preschool vision screening: rationale, methodology and outcome. Surv Ophthalmol 1996;41:3-30.

2. Ehrlich MI, Reinecke RD, Simons K. Preschool vision screening for amblyopia and strabismus. Programs, methods, guidelines, 1983. Surv Ophthalmol $1983 ; 28: 145-63$

3. Vinding T, Gregersen E, Jensen A, Rindziunski E. Prevalence of amblyopia in old people without previous screening and treatment. An evaluation of the present prophylactic procedures among children in Denmark. Acta Ophthalmol (Copenh) 1991;69:796-8.

4. Köhler L, Stigmar G. Vision screening of four-year-old children. Acta Paediatr Scand 1973;62:17-27.

5. Köhler L, Stigmar G. Visual disorders in 7-year-old children with and without previous vision screening. Acta Paediatr Scand 1978;67:373-7.

6. Bolger PG, Stewart-Brown SL, Newcombe E, Starbuck A. Vision screening in preschool children: comparison of orthoptists and clinical medical officers as primary screeners [commented on BMJ 1992;304:253; discussion p.253-4]. BMJ 1991;303:1291-4.

7. Newman DK, Hitchcook A, McCarthy H, Keast-Butler J, Moore AT. Preschool vision screening: outcome of children referred to the hospital eye service. Br J Ophthalmol 1996;80:1077-82.

8. Flom MC, Neumaier RW. Prevalence of amblyopia. Public Health Rep 1966; 81:329-41.
9. Temporini ER, Kara José N. Níveis de prevenção de problemas oftalmológicos. Arq Bras Oftalmol 1995;58:189-92.

10. TemporinI ER. Promoção da saúde ocular. Arq Bras Oftalmol 1999;62:82-4.

11. Jovanovic A. São Caetano: a revista/a survey. Fundação Pró-Memória. São Caetano do Sul, Cavassani Publicidade, 1997.

12. Costa MN, Kara José N, Machiaverni Filho N, Rangel FF, Rueda G, Pereira VL et al. Estudo da incidência de ambliopia, estrabismo e anisometropia em pré-escolares. Arq Bras Oftalmol 1979;42:249-52.

13. Abrahamsson M, Fabian G, Sjöstrand J. Refraction changes in children developing convergent or divergent strabismus. Br J Ophthalmol 1992;76:723-7.

14. Preslan MW, Novak A. Baltimore vision screening project. Ophthalmology 1996;103:105-9.

15. Friedman Z, Neumann E, Hyams SW, Peleg B. Ophthalmic screening of 38000 children, age 1 to $2 \frac{1}{2}$ years, in child Welfare clinics. J Pediatr Ophthalmol Strabismus 1980;17:261-7.

16. Scarpi MJ, José NK, Taiar A. Incidência de ambliopia em 1400 escolares da cidade de São Paulo, em 1975. Arq Bras Oftalmol 1977;40:16-23.

17. Oliver M, Nawaatzki, I. Screening of pre-school children for ocular anomalies. II-Amblyopia. Prevalence and therapeutic results at different ages. $\mathrm{Br}$ J Ophthalmol 1971;55:467-71.

18. Salomão SR, Ventura DF. Large sample population age norms for visual acuities obtained with Vistech-Teller acuity cards. Invest Ophthalmol Vis Sci 1995;36:657-70.

19. Brown EVL. Net average yearly changes in refraction of atropinized eyes from birth to beyond middle life. Arch Ophthalmol 1938;19:719-34.

20. Wood ICJ, Hodi S, Morgan L. Longitudinal change of refractive error in infants during the first year of life. Eye 1995;9(Pt 5): 551-7.

21. Ehrlich DL, Atkinson J, Braddick O, Bobier W, Durden K. Reduction of infant myopia: a longitudinal cycloplegic study. Vision Res 1995;35:1313-24.

22. Brown NP, Koretz JF, Bron AJ. The development and maintenance of emmetropia. Eye 1999;13(Pt 1):83-92.

23. Saunders K.J. Early refractive development in humans. Surv Ophthalmol 1995;40:207-16.

24. Abrahamsson M, Fabian G, Andersson K, Sjöstrand J. A longitudinal study of a population based sample of astigmatic children. I - Refraction and amblyopia. Acta Ophthalmol 1990;68:428-34.

25. Atkinson J, Braddick O, French J. Infant astigmatism measured by photorefraction. Science 1978;202:331-2.

26. Gwiazda J, Scheiman M, Mohindra I, Held R. Astigmatism in children: changes in axis and amount from birth to six years. Invest Ophthalmol Vis Sci 1984;25:88-92.

27. Ingram RM, Traynar MJ, Walker C, Wilsom JM. Screening for refractive errors at age 1 year: a pilot study. Br J Ophthalmol 1979;63:243-50.

28. Davidson DW. The future of vision screening. J Am Optom Assoc 1977;48: 469-76.

29. Ingram RM, Arnold PE, Dally S, Lucas J. Emmetropisation, squint, and reduced visual acuity after treatment. Br J Ophthalmol 1991;75:414-6.

\section{Ao enviarr unn anrtigo parra publicação,

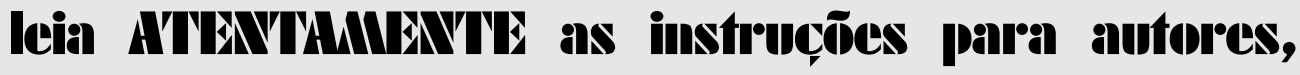 constante no final de cala fascículo.}

